\title{
Lipid-In, Sugar-Out: Early Results of Treatment with Additional Ketogenic Parenteral Nutrition in Left Ventricle Assist Device Patients.
}

Jonida Bejko

University of Padua

Tomaso Bottio ( $\nabla$ tbottio@gmail.com )

University of Padua

Raphael Caraffa

University of Padua

Massimiliano Carrozzini

University of Padua

Olimpia Bifulco

University of Padua

Vincenzo Tarzia

University of Padua

Dario Gregori

University of Padua

Demetrio Giorgio Pittarello

University of Padua

Francesco Francini Pesenti

University of Padua

Gino Gerosa

University of Padua

Francesco Tona

University of Padua

\section{Research Article}

Keywords: Right ventricle Failure, Ketogenic Parenteral Nutrition

Posted Date: August 4th, 2021

DOI: https://doi.org/10.21203/rs.3.rs-649111/v2 
License: (c) (i) This work is licensed under a Creative Commons Attribution 4.0 International License. Read Full License 


\section{Abstract}

Background and Aims: Right ventricle failure (RVF) can be a fatal complication following left ventricle assist device (LVAD) implant. Careful intra and postoperative management is becoming increasingly important. Unfortunately, the attempts are limited to the prediction and not to the treatment. There are no data on Ketogenic parenteral nutrition (KPN) for the targeted treatment of RVF. The aim of our retrospective observational case-control study is to determine whether KPN may play a role as additional treatment to conventional therapy after LVAD implantation.

Material and Methods: This is a retrospective single center analysis of a case series on prospectively collected data. From February 2012 to April 2021, 192 patients have been implanted with an LVAD. All the patients were managed with optimal medical and surgical therapy. One-hundred-seven patients were treated with additional KPN as adjuvant therapy against and in prevention of acute RVF (KPN-Group), defined according to International Society Heart and Lung Transplantation (ISHLT) guidelines. The remaining 85 patients, managed without KPN, constituted our control group (No-KPN-Group).

Results: In KPN-Group acute-RVF occurred in 31 patients (29\%), while causing death in 14 patients (13\%). In the No-KPN-Group acute-RVF was observed in 32 patients (37.6\%), while causing death in 20 patients (23.5\%). According to echocardiographic and hemodynamic results, the majority of KPN-Group patients responded with RV function improvement (range $4-60 \%$ of TAPSE increase, $p<0.05$ ) with recovery of RV function in $85 \%$ of the patients (91/107). In No-KPN-Group RV functional recovery was observed in 65 patients (76.4\%). Overall intra-hospital mortality was $23.4 \%$ (25) and $27.1 \%$ (23) in KPN-Group and NoKPN-Group, respectively $(p=0.337)$. While Intensive Care Unit $(I C U)$ stay was comparable $(p=0.078)$, wardstay was significantly shorter in KPN-Group $(p=0.03)$.

Conclusion: KPN was well tolerated. A complete recovery of the RV function was achieved in the majority of the patients and results lasted beyond the duration of KPN. Future research to understand the underlying mechanism, and identification of suitable patients and treatment duration is necessary.

\section{Introduction}

The metabolic requirements of the heart are the largest of any organ in the body. The main types of energy substrates for myocardial ATP synthesis are fatty acids (FFAs), carbohydrates, and ketone bodies. About $95 \%$ of ATP generated in the heart in normal conditions is derived from oxidative phosphorylation in the mitochondria and the remaining $5 \%$ from glycolysis and to a lesser extent from the Krebs cycle (1). In the healthy adult heart the mitochondrial oxidation of FFAs and glucose provides the majority of ATP production (2). At rest and in fasted state, approximately $70 \%$ to $90 \%$ of the acetylCoA which enters the citric acid cycle comes from the $\beta$-oxidation of FFAs, and the remaining $10-30 \%$ from the oxidation of pyruvate that is derived from glycolysis and lactate (3). Small amounts of ATP are also produced by oxidation of ketone bodies (KB) and amino acids. In early stages of left ventricular hypertrophy FFAs oxidation rate and the expression of FFAs oxidation enzymes are already decreased (4). 
It is generally assumed that the failing heart switches from mitochondrial oxidative metabolism toward glycolysis as a source of ATP production. In addition to the reduction in FFAs use, mitochondrial glucose oxidation can also decrease in the failing heart because of a decline in overall mitochondrial oxidative capacity and in the activity of the pyruvate dehydrogenase, the key rate-limiting enzyme of glucose oxidation (5).

Studies in animal models have recently showed that cardiac ketone oxidation is increased in the failing heart (6). Furthermore, plasma KB level raises in patients with severe heart congestive failure (7) and the role of $K B$ as an alternative fuel also in the failing human heart has been shown (8). Oxidation of $K B$ produces more ATP per oxygen consumed than does glucose.

The most effective way to provide KB to tissues is the ketogenic diet (KD). The KD is a high fat, low carbohydrate, and adequate protein diet. In the recent years the KD have been proposed as a potential and safe therapy for other conditions, such as neurologic disorders, mitochondrial disease and cancer (59).

As a consequence of a previous right heart dysfunction and/or due to post-operative right ventricle overload associated to post-operative anatomical modifications, the right ventricle failure (RVF) is the Achilles' heel of the mechanical assist devices therapy (10). Standardized specialized intensive care medical and surgical management are required. Unfortunately, several aspects of RVF following LVAD implant are still uncertain and careful intra and postoperative management is becoming increasingly important.

In this study we analyzed the in-hospital outcomes of 192 patients assisted by left-ventricular-assistdevice (LVAD) with HF for different etiology, depending on whether they were managed with (107 patients) or without (85 patients) the Ketogenic Parenteral Nutrition (KPN) as additional medical therapy.

\section{Materials And Methods}

The local ethics committee approved review of the data, consented and approved the retrospective analysis on prospectively collected data (IRB number 34459, 05/26/2021). The patient informed consent was obtained from all subjects specifying the type of adjuvant parenteral nutrition. Medical history, daily progress notes, diet information, relevant laboratory data, imaging findings, and complications were prospectively updated.

Primary endpoints were to understand the impact of this adjuvant treatment on the clinical outcomes and on RV function after LVAD implant. Differences in echocardiographic parameters as RV Ejection Fraction (RVEF), fractional Area Change (FAC), Tricuspid Annular Plain Systolic Excursion (TAPSE), Global longitudinal Strain (GLS), were recorded before and after KPN. The secondary end-points were identifying effects on urine output volume, liver and kidney laboratory tests, glycemic variability (GV), and the occurrence of adverse events before and after KPN. Additional secondary end-points were clinical 
outcomes, such as post-operative intensive care unit (ICU) stay, word stay and intra-hospital mortality in LVAD patients with or without adjuvant treatment with KPN.

From February 2012 to April 2021, 192 patients have been implanted with an LVAD (HeartWare 54, Jarvik 62, HMIII 76). Post-implant acute RVF, defined according to International Society Heart and Lung Transplantation (ISHLT) guidelines, occurred in 63 patients (32.8\% RVF incidence). Totally, 107 patients, were treated with additional KPN as adjuvant therapy against and or in prevention of acute RVF (KPNGroup). KPN was introduced as additional treatment soon after Hospital admission in 69 patients (38 patients with temporary ECLS and 31 patients with poor RV contractility at echocardiographic parameters) and soon after LVAD implant in 38 patients, as a choice of their first operator. Exclusion criteria for adjuvant treatment were the following: diabetes type 1, hereditary or acquired disorders of triglyceride hydrolysis, severe metabolic acidosis, impaired lipid utilization, or severe coagulopathy, as contraindicated to lipid emulsion therapy (11). The remaining 85 patients, managed without KPN, constituted our control group (No-KPN-Group).

\section{Diet protocol.}

The KPN provided a mixed aminoacid solution (Sintamin10\%, Fresenius Kabi, Bad Homburg, Germany) at the dose of $1 \mathrm{~g}$ of aminoacid per kilogram of body weight per day and a $20 \%$ lipid emulsion (SMOF-lipid $20 \%$, Fresenius-Kabi, Bad Homburg, Germany) at the dose of 1,8 $\mathrm{g}$ of lipid per kilogram of body weight per day. The lipid emulsion contained soybean oil, olive oil, medium chain triglycerides (MCTs), fish oil and glycerol $(25 \mathrm{~g} / \mathrm{l})$. Electrolytes, multivitamin supplement (Cernevit, Baxter SPA, Rome, Italy) and trace elements (Olitrace, B. Braun, Melsungen, Germany) were infused daily in $0,9 \%$ sodium chloride solution. Treatment was used as an intravenous infusion until complete oral feeding and then continued orally until purpose was achieved. Therefore it was started while in the ICU and on the ward, and then continued once at home. The treatment would be stopped at any time in case of intolerance of any kind.

In our KPN protocol we did not add glucose because of the small amount of glycerol (12,5 g per day), a gluconeogenic precursor, given with the lipid emulsion. Daily energy infusion, approximately 1300 kilocalories per day, did not meet the theoretical requirements in most subjects. This energy provision was chosen to avoid the infusion of too large amounts of lipids and considering the short period of endovenous nutrition decided to apply.

\section{Biochemical analysis.}

During the treatment plasma triglycerides, urea, creatinine, ALT (alanine transferase), AST (aspartate transferase) were evaluated according to the scheme in Table I. Blood glucose was measured using ACCU-CHECK Inform II BG monitoring system (Roche Diagnostic, Roche Rotkreuz, Switzerland). Capillary beta-hydroxy-butyrate (BHB) was measured using electrochemical method (FreeStyle Optium H ketone, Abbott Diabetes Care Ltd., Oxon, UK). Urinary KBs were measured by testing strips Siemens Multistics 10 
SG (Siemens Healthcare Diagnostics, Deerfield, IL, USA). The assays were conducted according to the manufacturer's instructions. The glycemic control protocol was performed as previously described (maintaining serum glucose levels $120-180 \mathrm{mg} / \mathrm{dL}$ ) (12). Glucose variability (GV) was calculated using standard deviation (SD) of blood glucose measurements.

\section{The echocardiography:}

exams were performed in blind by the cardiologists, before treatment with KPN, at the $6^{\text {th }}$ day of administration and then every six further days of treatment. The parameters considered to evaluate RV function were fractional area change (FAC \%), RV ejection fraction (RVEF \%), tricuspid annulus peak systolic velocity (TAPSE) (mm), Global longitudinal strain rate $\left(\mathrm{s}^{-1}\right)$, pulsed Doppler RV index of myocardial performance (RIMP), tissue Doppler RIMP, tissue Doppler $\mathrm{S}^{\prime}$ wave (cm/s), color tissue Doppler $\mathrm{S}$ wave $(\mathrm{cm} / \mathrm{s})$ and $\mathrm{dP} / \mathrm{dT}(\mathrm{mmHg} / \mathrm{s})$. The RVF was defined according to the ISHLT annual INTERMACS reports (13).

\section{RVF medical management and indications for $R V$ support:}

RV support was indicated in: (i) patients who suffered overt RV failure during or immediately after weaning from cardiopulmonary bypass despite maximal medical management according to the ISHLT guidelines (14) and (ii) patients at high risk of postimplant RV failure like patients on extracorporeal life support (ECLS) prior to LVAD implantation. Indeed, due to the difficulty of RV assessment in these patients, they were considered at high risk and received systematic RV support after LVAD implantation.

\section{Criteria for weaning RV support:}

Patients were evaluated daily for RV recovery by collecting haemodynamic parameters, routine blood tests and by performing weaning trials during which the RVAD flow was reduced to $\sim 1.5 \mathrm{I} / \mathrm{min}$ for 15 min. $R V$ recovery was defined as a return to the baseline values of the echocardiographic parameters. The following criteria were retained for weaning of RV support: (i) low levels of inotropic support (dobutamine $<5$ y kg-1.min-1; epinephrine or norepinephrine $<0.05$ y kg-1.min-1); (ii) low lactate levels ( $<2.1 \mathrm{mmol} / \mathrm{L}$ ) and (iii) stable mean systemic arterial blood pressure around $70 \mathrm{mmHg}$, with no RV dilatation at echocardiography and no decrease in LVAD flow during weaning trials. Central venous pressure or other right-sided haemodynamic parameters were not considered (right heart catheterization), since their interpretation in the patients under partial RVAD support is awkward.

\section{Statistical analyses}

Continuous variables are expressed as mean \pm standard deviation. To analyze trends in observed variables, a linear regression model was estimated using a linear spline transform of time, for evaluating 
simultaneous trend and change in slope effects of the trajectories over time of each of the variables considered. Significance of trends and change in slope at time of treatment initiation were evaluated using the Akaike Information Criterion for the linear (trend) and non-linear (change in slope at "zero" time in the linear spline). Analyses were performed using the R System (15) and the gMCP and rms (16) Libraries.

\section{Results}

KPN-Group and No-KPN-Group patients' demographics are reported in table II. The preoperative patients characteristics were comparable through the groups. All patients were naive to diet therapy at the time of beginning the KPN, they never taken this type of diet before. The majority was affected by dilated cardiomyopathy (DCM) secondary to ischemic pathology (48-49\%), while it was primary DCM in the remaining $46-43 \%$ of the population. I-III INTERMACS profiles were predominant (75-78\%). Pre-operative temporary mechanical circulatory support (t-MCS) was necessary in $35 \%$ of patients in KPN-Group while it was present in $29 \%$ of No-KPN-Group $(p=0.350)$. The intention to treat strategy was comparable through the groups.

Post-operative outcomes are reported on Table III. Conventional medical and surgical post-operative care were comparable. All patients were treated with inotropic infusion ( $3 \pm 1$ different drugs). Diuretics were administered in continuous infusion in $85 \%$ and $89 \%$ of the patients; while in $31 \%$ and $38 \%$ continuous veno-venous hemofiltration (CVVH) was necessary in KPN and No-KPN groups, respectively. Continuous intravenous administration of insulin was necessary in $35 \%$ and $39 \%$ of the population. KPN was administered for $16 \pm 6$ days.

Acute-RVF occurred in 31 (29\%) and 32 (38\%) patients in KPN-Group and No-KPN-Group, respectively $(p=0.219)$. RVAD implantation was necessary in $19(18 \%)$ and $23(27 \%)$ patients in KPN-Group and NoKPN-Group, respectively $(\mathrm{p}=0.078)$. RVF-related death was higher in No-KPN-Group ( $13 \%$ vs $24 \%)$, although not significant $(p=0.06)$. Out of patients assisted with post-operative RVAD, 4 patients $(21 \%)$ died in KPN-Group while 12 patients (52\%) died in No-KPN-Group ( $\mathrm{p}=0.05)$. Overall intra-Hospital mortality was comparable occurring in 25 patients in KPN-Group (23\%) and 23 patients in No-KPN-Group (27\%). Cerebral events, cerebral and systemic hemorrhagic events caused death in 17 patients in KPN-Group $(16 \%)$ and 3 patients in No-KPN-Group (4\%). Mean ICU-stay $(p=0.078)$ and mean ward-stay $(p=0.003)$ were higher in No-KPN-Group, while resulting significantly longer only the mean ward-stay.

Pre-KPN and post-KPN biochemistry results are reported on Table IV. Increase in mean serum triglyceride level was observed $(p<0.05)$ after KPN administration. The treatment was suspended for occurrence of very high hyperlipemia (triglyceride level $>5.6 \mathrm{mml} / \mathrm{L}$ ) in $5 \%$ of the patients (5). All these patients died for multi-organ-failure (MOF) as a consequence of acute-RFV without recovery. KPN was suspended in 2 additional patients who underwent heart transplant: 1 for recurrent malignant arrhythmias and the second for un-manageable recurrent RVF. Plasma urea and creatinine levels were stable during the KPN treatment, as well as ALT, AST, and total bilirubin levels (Table IV). 
On the sixth day urine test strips were positive for ketonuria in $92 \%$ of patients, ranging from +1 to +2 . Beta-hydroxy-butyrate (BHB) plasma levels after six day exceeded the normal range in all subjects (mean $2.06 \pm 0.9 \mathrm{mmol} / \mathrm{l})$ (Table IV).

At echocardiographic exams, excluding 14 patients who died for MOF and 2 patients heart transplanted, all KPN-Group patients improved RV parameters (Table V). The inotropic requirements changed after $3 \pm 1$ days from diet onset, with reduction and progressive suspension of the support $15 \pm 7$ days. We collected the data of LV ejection fraction as well, with improvement of the function from $30 \pm 15 \%$ to $37 \pm 17 \%$ $(p<0.01)$.

We further compared the trends of glycaemia, glycaemia variability (GV), urine output volume, insulin and diuretics posology in the first three days after administration of KPN, with the treatment period (from $1^{\text {st }}$ to $6^{\text {th }}$ day), as the supposed time of onset of ketosis is of almost 3-5 days of treatment.

The glucose levels were maintained stable, in both, first 3 days and during KPN-treatment $(p=0.45)$. Anyway, significant trend in reduction during KPN was observed $(p<0.001)$ (Figure 1), although insulin doses necessary to maintain the range did not show differences ( $p$-value for change at day " 0 ” 0.41 ; overall linear trend $p=0.12)$. Accordingly, the $G V$ reduced significantly $(p=0.034)$ in linear trend $(p<0,001)$ (Figure 2). Increase in urine output volume was observed in patients with preserved diuresis, treated with KPN, but not statistically significant (Figure 3), despite similar diuretics posology in pre and during KPNperiod ( $p$-value for change in trend at day " 0 " 0.79 ; overall linear trend $p=0.35$ ). As far as liver function concerns, we did not observe statistically significant differences between before and after treatment with KPN (Table IV).

\section{Discussion}

In this study we present the clinical outcomes of 191 patients assisted by LVAD. One-hundred and seven patients were managed with KPN as additional treatment during in-hospital stay. Their results were compared to 85 patients who were managed by only optimal medical and surgical therapy. Our current results may expand the indications of an already confirmed therapy.

Heart transplantation is considered the gold-standard therapy for end-stage HF, but is limited by insufficient donor supply. At present, implantable LVADs represent the only available and effective alternative to heart transplantation. However, given that LVADs support only the LV, after device implantation RV is exposed to the risk of developing severe dysfunction $(17,18)$. In addition, RV hemodynamics changes after LVAD implantation because RV output must match that of the flow generated by the device (19). RVF can be a fatal complication that requires specialized intensive care management and portends a worse prognosis in the short as well as the long term (20). Unfortunately, several aspects of RVF following LVAD implant are still uncertain, and even the reported prevalence of RVF in this population has been variable (ranging between $13 \%$ and $51 \%$ ) (13). Careful intra and 
postoperative management is becoming increasingly important (20). Currently, with regard to this painful Achilles' heel, the attempts are limited to the prediction and not to the treatment (21).

The KD is a high fat, low carbohydrate, and adequate protein diet. Guelpa and Marie introduced KD in France 1911 but Wilder at the Mayo-clinic in 1921 was the first to use KD as a treatment for epilepsy (5).

Our strategy was inspired by two studies $(1,3)$. Aubert et al. (1), demonstrated that the proteins required for cellular FFAs utilization were reduced in myocardial samples from both hypertrophied and failing mice hearts. Meanwhile, gene and protein expression of BDH1 was elevated (1). Bedi et al. (3), in a contemporary study, aimed to elucidate the metabolic signature in the human non-diabetic failing heart. They concluded that their results support the role of KB as an alternative fuel and myocardial ketone oxidation as a key metabolic adaption in the failing human heart. In end-stage HF, insulin resistance and cardiac cachexia are common, which increases the likelihood of ketone production by the liver and renders cardiac ketone utilization unavoidable (2).

Based on the recommendations of food intake for healthy persons and by clinical experience, it is recommended that parenteral lipids should usually provide about $25-40 \%$ of non-protein calories, depending on the individual patient's tolerance to carbohydrates and lipids. In severe compromised patients, a higher lipid intake of up to $50 \%$ of the non-protein calories may be advisable (22). Lipid infusion significantly increased LV systolic pressure in animal ex-vivo models (4). In humans, isolated case reports have reported improvement of cardiomyopathies after high-fat diet (23).

In accordance with the first operator and with our specialist in nutrition (FFP) choice, we treated with KPN 107 LVAD patients (KPN-Group). The population treated with mechanical support in our center is particularly ill, considering the $2 / 3$ of patients in INTERMACS profiles I-III and the high percentage of patients requiring $\mathrm{CVVH}$ and or mechanical support with t-MCS. This was one of the reasons why we decided to test this therapy in addition to the conventional ones. All these patients were already treated with the conventional therapies used to assist failing heart in clinical practice. Eighty-five patients were exclusively managed by optimal medical and surgical therapy (No-KPN-Group). Overall, RVF incidence in our population was 33\% (63/192). RVF incidence was higher in the no-KPN-group (29\% vs $37.6 \%$ ). Although the results were not significantly different depending on whether KPN was used or not, we observed higher mortality, higher RVF-related mortality, longer ICU and ward lengths of stay when KPN was denied. While considering that the clinical results have not significantly changed, it is possible to stress the following point: hospitalization time increases relative costs, against a minimum increase in costs related to the use of the KPN. In KPN-Group, while considering the cardiac function at echocardiogram, we observed an excellent response of the RV with significant improvement, in correspondence to the increase of KB. RV systolic function is a reflection of contractility, afterload, and preload. The increase in urine output observed, although not significant, may be part of the mechanism that improved RV function. Our attention was particularly attracted by the differences recorded in the echo parameters as RVEF, FAC, TAPSE, GLS strain rate. Although those parameters normally should be viewed as a general indication of load dependence, the significance of the changes observed cannot be 
attributed to this only variation. Of particular interest may be the more relevant increase in the strain rates of basal and apical sections of the RV, versus the mid ones (24-25).

As myocardial metabolic abnormalities have been predominantly described in tissue samples from the LV $(1,2)$, we collected the data on ejection fraction variations during KPN. Improvement was observed even in LV function, but considering the population under investigation, the data need to be confirmed with further studies.

High GV was demonstrated strongly related to increasing mortality in heterogeneous population of critically ill patients, in short and long-term follow-up (26-27). In both, pre and during KPN, we maintained stable glucose levels, in accordance to the Society of Thoracic Surgeons Practice Guidelines (12). Throughout all the period of treatment, in our population the GV was significantly reduced, without differences in hypoglycemic treatment.

The most frequent collateral effect was the increase in plasma triglyceride levels; values of approximately $4.6 \mathrm{mmol} / \mathrm{l}(400 \mathrm{mg} / \mathrm{dl})$ can be physiologically reached post-prandial and are considered acceptable during infusion of lipid emulsions. Very high pathologic levels instead, were recorded in 5 patients in which MOF was already ongoing. In these patients, the already established organ damage subsequently led to death. Despite improved RV function, complete recovery was not possible in all patients.

To date, the majority of INTERMACS enrollees have the most severe degrees of hemodynamic compromise (profiles I, II), which are associated with the highest 12-month post-implant mortality (13). The INTERMACS Annual Report confirms a shift to implantation in earlier stages of HF with a decrease in proportion of profile 1 patients from $35 \%$ to $17 \%$ in the contemporary era (13). Unfortunately, surgeons still have a high percentage of low INTERMACS profiles presented as candidates to LVAD implantation. RVF following LVAD implantation is a vexing clinical problem characterized by low VAD flows and systemic hypoperfusion despite elevated right-sided filling pressures. Thus, it is critical that implanting centers advance attempts not only limited to the prediction but also to the treatment of right heart function. Improvements in nutritional parameters can favorably impact postoperative outcomes.

\section{Limitations}

The main limitation of this clinical study is that it is a retrospective study. We tried to improve it by adding a case-control group. The entity of contribution on the improvement in RV function of the KPN and the concomitant therapies need to be assessed. The increase in serum lipids (triglycerides) due to KPN can also have a confounding meaning, even if it is an intrinsic part of the hyperlipidic therapy itself. We are conducting further analyses to understand the underlying mechanism and the outcomes at medium and long-term effects of the treatment.

\section{Conclusions}


$\mathrm{KPN}$ was safe and well tolerated. A RV recovery function was achieved in the majority of the patients. Further studies are mandatory to understand the underlying mechanism and better define the indications and timing to this treatment.

\section{Declarations}

\section{Ethics approval}

The local ethics committee (Azienda Ospedaliera di Padova, Italy) approved review of the data, consented and approved the retrospective analysis on prospectively collected data (IRB number 34459, $05 / 26 / 2021)$. We confirm that all methods were carried out in accordance with relevant guidelines and regulations

\section{Consent for publication}

Not applicable

\section{Availability of data}

The datasets used and/or analysed during the current study are available from the corresponding author on reasonable request.

\section{Competing Interests'}

All authors have no conflicts of interest or financial conflicts to disclose.

\section{Funding}

No funding source was provided for this study.

\section{Author contribution}

$\mathrm{JB}$ data analysis and writing original version; TB data analysis and original version reviewer; RC dataset updating; $\mathrm{MC}$ dataset updating; $\mathrm{OB}$ dataset updating; VT assisting data analysis; DG formal data analysis; DGP; echocardiographic data analysis; FFP study conceptualization; GG study conceptualization; FT study conceptualization. 


\section{References}

1. Aubert, G. et al. The Failing Heart Relies on Ketone Bodies as a Fuel., 23 (8), 698-705 (2016 Feb).

2. Lommi, J., Koskinen, P., Näveri, H., Härkönen, M. \& Kupari, M. Heart failure Ketosis. Journal of Internal Medicine, 242, 231-238 (1997).

3. Bedi KCJr, Snyder, N. W. et al. Evidence for Intramyocardial Disruption of Lipid Metabolism and Increased Myocardial Ketone Utilization in Advanced Human Heart Failure. Circulation. 2016 Feb 23;133(8):706 - 16.

4. Park, J. et al. Lipofundin $\circledast$ MCT/LCT $20 \%$ increase left ventricular systolic pressure in an ex vivo rat heart model via increase of intracellular calcium level. Korean J Anesthesiol, 69 (1), 57-62 (2016 Feb).

5. Stafstrom, C. E. Dietary approaches to epilepsy treatment: old and new options on the menu. Epilepsy Curr. 2004 Nov-Dec;4(6):215 - 22.

6. Liu, Y. M. \& Wang, H. S. Medium-chain triglyceride ketogenic diet, an effective treatment for drugresistant epilepsy and a comparison with other ketogenic diets. Biomed J, 36 (1), 9-15 (2013).

7. Pfeifer, H. H., Lyczkowski, D. A. \& Thiele, E. A. Low glycemic index treatment: implementation and new insights into efficacy.Epilepsia2008;49 Suppl 8:42 - 5

8. Jung, D. E., Kang, H. C., Lee, J. S., Lee, E. J. \& Kim, H. D. Safety and role of ketogenic parenteral nutrition for intractable childhood epilepsy Brain Dev. 2012 Sep;34(8):620-4.

9. Branco, A. F. et al. et al. Ketogenic diets: from cancer to mitochondrial diseases and beyond. Eur J Clin Invest, 46 (3), 285-298 (2016 Mar).

10. Lampert, B. C. \& Teuteberg, J. J. Right ventricular failure after left ventricular assist devices. J Heart Lung Transplant, 34 (9), 1123-1130 (2015 Sep).

11. Adolph, M. et al. Working group for developing the guidelines for parenteral nutrition of The German Association for Nutritional Medicine. Lipid emulsions - Guidelines on Parenteral Nutrition, Chap. 6.Ger Med Sci. 2009 Nov 18;7:Doc22.

12. Mc Donnell, M. E., Alexanian, S. M., Junqueira, A., Cabral, H. \& Lazar, H. L. Relevance of the Surgical Care Improvement Project on glycemic control in patients undergoing cardiac surgery who receive continuous insulin infusions. J Thorac Cardiovasc Surg, 145 (2), 590-594 (2013).

13. Kirklin, J. K. et al. Seventh INTERMACS annual report: 15,000 patients and counting. J Heart Lung Transplant 2015;34:1495 - 504.

14. Slaughter, M. S. et al. et al. HeartMate II Clinical Investigators. Clinical management of continuousflow left ventricular assist devices in advanced heart failure. J Heart Lung Transplant, 29 (4 Suppl), S1-39 (2010 Apr).

15. R Development Core Team. $R$ : A language and environment for statistical computing (R Foundation for Statistical Computing, Vienna, Austria, 2015).

16. Harrell, F. E. J. rms: Regression Modeling Strategies. R package version 4.1-32014. Available from: http://CRAN.R-project.org/package=rms. 
17. Bejko, J., Toto, F., Gregori, D., Gerosa, G. \& Bottio, T. Left ventricle assist devices and driveline's infection incidence: a single-centre experience. J Artif Organs, 21 (1), 52-60 (2018 Mar).

18. Bejko, J., Pittarello, D. \& Falasco, G. et al. A pilot study on the efficacy and safety of a minimally invasive surgical and anesthetic approach for ventricular assist device implantation. Int $J$ Artif Organs, 41, 28-36 (2018). Di Gregorio GTarzia V, Rizzoli G

19. Vignati, C., Apostolo, A., Cattadori, G. \& Farina, S. et al. Lvad pump speed increase is associated with increased peak exercise cardiac output and vo2, postponed anaerobic threshold and improved ventilatory efficiency. Int J Cardiol, 1;230, 28-32 (2017 Mar). Del Torto AScuri S

20. Carrozzini, M. et al. et al. Results of new-generation intrapericardial continuous flow left ventricular assist devices as a bridge-to-transplant. J Cardiovasc Med (Hagerstown), 19 (12), 739-747 (2018 Dec).

21. Soliman, O. I. I. et al. EUROMACS Investigators. Derivation and Validation of a Novel Right-Sided Heart Failure Model After Implantation of Continuous Flow Left Ventricular Assist Devices: The EUROMACS (European Registry for Patients with Mechanical Circulatory Support) Right-Sided Heart Failure Risk Score. Circulation 2018 Feb 27;137(9):891-906

22. Gormsen, L. C. et al. et al. Ketone Body Infusion With 3-Hydroxybutyrate Reduces Myocardial Glucose Uptake and Increases Blood Flow in Humans: A Positron Emission Tomography Study. J Am Heart Assoc, 6 (3), e005066 (2017 Mar).

23. Francini-Pesenti, F., Tresso, S. \& Vitturi, N. Modified Atkins ketogenic diet improves heart and skeletal muscle funcion in glycogen storage disease type III. Acta Myol, 38(1) (1), 17-20 (2019 Mar).

24. Carluccio, E. et al. et al. Superior Prognostic Value of Right Ventricular Free Wall Compared to Global Longitudinal Strain in Patients With Heart Failure. J AM Soc Echocardiogr, 32, 836-844 (2019).

25. Borovac, J. A. et al. et al. Right Ventricular Free Wall Strain and Congestive Hepatopathy in Patients With Acute Worsening of Chronic Heart Failure: A CATSTAT-HF Echo Substudy. J Clin Med, 9, E1317 https://doi.org/10.3390/jcm9051317 (2020). doi

26. Capes, S. E., Hunt, D., Malmberg, K. \& Gerstein, H. C. Stress hyperglycaemia and increased risk of death after myocardial infarction in patients with and without diabetes: A systematic overview., 355, 773-778 (2000).

27. Krinsley, J. S. Association between hyperglycemia and increased hospital mortality in a heterogeneous population of critically ill patients. Mayo Clinic Proc, 78, 1471-1478 (2003).

\section{Tables}

Table I

Scheme of examinations performed during the diet treatment. 


\begin{tabular}{|ll|}
\hline Parameter & KPN \\
\hline Serum Glucose & 8 times/daily \\
\hline Serum Triglycerides & 2 times/week \\
\hline Serum urea & Once daily \\
\hline Serum creatinine & Once daily \\
\hline ALT, AST & 2 times/week \\
\hline Urine KB & Once daily \\
\hline Capillary BHB & Once daily \\
\hline Echocardiography & Baseline- every 6 days of treatment \\
\hline $\begin{array}{l}\text { KB- ketone bodies; KPN- ketogenic parenteral nutrition, BHB - beta-hydroxy-butyrate; ALT- alanine } \\
\text { transferase, AST- aspartate transferase. }\end{array}$ \\
\hline
\end{tabular}

Table II

Baseline patient's characteristics 
Variable

Age (years)
Sex (male)
BSA $\left(\mathrm{m}^{2}\right)$
Preop t-MCS

\section{Etiology:}

DCM as evolution to ICM

DCM due to Valvulopathy

Primitive DCM

Arrhythmogenic Cardiomyopathy

\section{Pre-LVAD LV-parameters:}

$\operatorname{LVEDD}(\mathrm{mm})$
$\operatorname{LVESD}(\mathrm{mm})$
$\operatorname{LVEDV}(\mathrm{ml} / \mathrm{mq})$
$\operatorname{LVEF}(\%)$

Intention to treat:

BTT strategy (Bridge to Transplant)

BTC strategy (Bridge to Candidacy)

DT strategy (Destination Therapy)

INTERMACS profile:

I-III

IV

\section{Hemodynamic variables:}

Mean PA (mmHg)

Mean RA (mmHg)

PCWP (mmHg)

RA/PCWP

TPG (mmHg)
KPN-Group ( $n=107)$

No-KPN-Group ( $\mathrm{n}=85$ )

p

$58.3 \pm 11.2$

$59.2 \pm 14.0$

0.631

$84.1 \%(90)$

$90.6 \%(77)$

0.203

$1.89 \pm 0.21$

$1.84 \pm 0.25$

0.178

$35.5 \%(38)$

$28.2(24)$

0.350

0.896

$48.6 \%(52)$

$49.4 \%(42)$

$2.8 \%(3)$

$4.7 \%(4)$

$45.8 \%(49)$

$43.5 \%(37)$

$2.8 \%(3)$

$2.4 \%(2)$

$69.6 \pm 12.8$

$69.4 \pm 12.6$

0.729

$64.4 \pm 13.4$

$64.2 \pm 13.6$

0.657

$140.7 \pm 45.1$

$135.0 \pm 46.0$

0.829

$20.5 \pm 5.8$

$22.5 \pm 5.9$

0.368

0.060

$32.9 \%$ (28)

$30.6 \%$ (26)

$36.5 \%$ (31)

$22.4 \%$ (24)

0.607

$74.8 \%(80)$

$78.8 \%(67)$

$25.2 \%(27)$

$21.2 \%(18)$ 


\begin{tabular}{lllc}
$\mathrm{Cl}(\mathrm{I} / \mathrm{min} / \mathrm{m} 2)$ & $1.81 \pm 0.47$ & $1.91 \pm 0.70$ & 0.313 \\
\hline $\mathrm{PVR}(\mathrm{wood})$ & $4.4 \pm 2.8$ & $4.5 \pm 3.0$ & 0.457
\end{tabular}

\section{Laboratory variables:}

\begin{tabular}{llll}
\hline Hct $(\%)$ & $31.6 \pm 7.5$ & $31.4 \pm 7.8$ & 0.451 \\
\hline Plat $(\mathrm{x} 103 / \mathrm{ml})$ & $229 \pm 98$ & $216 \pm 107$ & 0.390 \\
\hline $\mathrm{Na}(\mathrm{mEq} / \mathrm{l})$ & $137.7 \pm 5.4$ & $137.1 \pm 6.3$ & 0.428 \\
\hline Crea $(\mathrm{mg} / \mathrm{dl})$ & $1.59 \pm 1.03$ & $1.42 \pm 0.73$ & 0.294 \\
\hline Alb $(\mathrm{mg} / \mathrm{dl})$ & $3.8 \pm 0.5$ & $3.6 \pm 0.8$ & 0.391 \\
\hline T-Bil $(\mathrm{mg} / \mathrm{dl})$ & $1.59 \pm 2.15$ & $1.26 \pm 1.34$ & 0.211 \\
\hline
\end{tabular}

DCM - dilative cardiomyopathy; ICM - ischemic cardiomyopathy; RV - right ventricle; LVAD - left ventricle assist device; RVAD - right ventricle assist device; Alb - albumin; $\mathrm{Cl}$ - cardiac index; Crea creatinine; Hct - hematocrit; $\mathrm{Na}$ - sodium; PA - pulmonary artery; PCWP - pulmonary capillary wedge pressure; Plat - platelet count; PVR - Pulmonary vascular resistance; RA - right atrial (pressure); RVF right ventricular failure; T-Bil - total bilirubin; TPG - transpulmonary gradient. CVVH - continuous venous-venous hemofiltration; KPN - ketogenic parenteral nutrition; Mean \pm standard deviation.

Table III

Post-operative outcomes. 


\begin{tabular}{|c|c|c|c|}
\hline Conventional therapies: & $\begin{array}{l}\text { KPN-Group } 107 \\
\text { pts }\end{array}$ & $\begin{array}{l}\text { No-KPN-Group } 85 \\
\text { pts }\end{array}$ & $\mathbf{p}$ \\
\hline LVAD & $100 \%(107)$ & $100 \%(85)$ & \\
\hline Post-op RVAD & $17.8 \%(19)$ & $27.1 \%(23)$ & 0.078 \\
\hline Inotropic infusion & $100 \%(107)$ & $100 \%(85)$ & \\
\hline Diuretics infusion & $85 \%(91)$ & $89 \%(76)$ & 0.654 \\
\hline $\mathrm{CVVH}$ & $30.8 \%(33)$ & $37.6 \%(32)$ & 0,359 \\
\hline Insulin Infusion & $35 \%(37)$ & $39 \%(33)$ & 0.541 \\
\hline \multicolumn{4}{|l|}{ Ventilation management } \\
\hline Intubation length (days) with NO therapy & $3.2 \pm 7.9$ & $3.7 \pm 5.3$ & 0.646 \\
\hline Tracheostomy & $2.8 \%(3)$ & $14.1 \%(12)$ & 0.005 \\
\hline Type of LVAD: & & & $<0.001$ \\
\hline Heartmate III & $24.3 \%(26)$ & $58.8 \%(50)$ & \\
\hline Heartware & $35.5 \%(38)$ & $18.8 \%(16)$ & \\
\hline Jarvik 2000 & $40.2 \%(43)$ & $22.4 \%(19)$ & \\
\hline \multicolumn{4}{|l|}{ LVAD speed: } \\
\hline Heartmate III & $5500 \pm 100 \mathrm{rpm}$ & $5500 \pm 100 \mathrm{rpm}$ & \\
\hline Heartware & $4500 \pm 100 \mathrm{rpm}$ & $4500 \pm 100 \mathrm{rpm}$ & \\
\hline Jarvik 2000 & $3 \pm 1$ & $3 \pm 1$ & \\
\hline Acute RVF & $29.0 \%(31)$ & $37.6 \%(32)$ & 0,219 \\
\hline RVF related death & $45.2 \%(14)$ & $62.5 \%(20)$ & 0,060 \\
\hline Days of KPN & $16 \pm 6$ & - & \\
\hline \multicolumn{4}{|l|}{$\begin{array}{l}\text { Causes of KPN interruption before RV } \\
\text { recovery: }\end{array}$} \\
\hline Very high Hyperlipemia & $4.6 \%(5)$ & - & \\
\hline Heart transplantation & $1.9 \%(2)$ & $1.1 \%(1)$ & \\
\hline ICU stay & $13 \pm 17$ & $18 \pm 17$ & 0,078 \\
\hline Ward Stay & $22 \pm 17$ & $36 \pm 32$ & 0,003 \\
\hline Intra-hospital Mortality & $23.4 \%(25)$ & $27.1 \%(23)$ & 0,337 \\
\hline
\end{tabular}




\section{Table IV}

KPN serum and urine analyses (values are reported as mean $\pm S D$ ).

\begin{tabular}{|c|c|c|c|}
\hline & Pre-KPN & Post-KPN (from the $6^{\text {th }}$ day) & p-value \\
\hline TG (mmol/l) & $1.06 \pm 0.43$ & $3.13 \pm 3.55$ & $<0.05$ \\
\hline Urea (mmol/l) & $10.27 \pm 8.03$ & $10.78 \pm 7.91$ & 0.82 \\
\hline Creatinine (umol/l) & $94.09 \pm 59.55$ & $110 \pm 48.49$ & 0.43 \\
\hline ALT (U/I) & $53.4 \pm 5.5$ & $53.8 \pm 6.6$ & 0.88 \\
\hline AST (U/I) & $40.9 \pm 8.7$ & $44.4 \pm 11.9$ & 0.46 \\
\hline T-Bilirubin (mg/dl) & $1.5 \pm 0.8$ & $1.4 \pm 0.5$ & 0.5 \\
\hline Urine Ketone Bodies (mmol/l) & 0 & $1.81 \pm 2.52$ & $<0.05$ \\
\hline Capillary BHB (mmol/l) & 0 & $2.06 \pm 0.9$ & $<0.001$ \\
\hline \multicolumn{4}{|c|}{$\begin{array}{l}\text { KPN- ketogenic parenteral nutrition; From the } 6^{\text {th }} \text { day of treatment- Biochemical assessments were } \\
\text { performed starting from the sixth day and then every two days until the discontinuation of therapy } \\
\text { and / or home discharge; TG- plasma triglycerides; ALT- alanine transferase, AST- aspartate } \\
\text { transferase; BHB- beta-hydroxy-butyrate. }\end{array}$} \\
\hline
\end{tabular}

Table V

Echocardiography data 


\begin{tabular}{|lllc|}
\hline RV Parameters & Pre KPN (mean \pm SD) & Post-KPN (mean \pm SD) & p-value \\
\hline FAC (\%) & $31 \pm 5$ & $40 \pm 7$ & $<0.05$ \\
\hline RVEF (\%) & $30 \pm 3$ & $37 \pm 6$ & $<0.05$ \\
\hline Global longitudinal Strain rate $\left(\mathrm{s}^{-1}\right)$ & & & $<0.05$ \\
Basal & $1 \pm 0.38$ & $1.5 \pm 0.38$ & \\
Mid & $1.2 \pm 0.41$ & $1.3 \pm 0.46$ & \\
Apical & $1.6 \pm 0.27$ & $2.01 \pm 0.03$ & $<0.05$ \\
\hline TAPSE (mm) & $9 \pm 2$ & $13 \pm 2$ & 0.06 \\
\hline Pulsed Doppler RIMP & $0.5 \pm 0.085$ & $0.35 \pm 0.09$ & $<0.05$ \\
\hline Tissue Doppler RIMP & $0.6 \pm 0.08$ & $0.42 \pm 0.07$ & 0.08 \\
\hline Tissue Doppler S' wave (cm/s) & $8.2 \pm 1.3$ & $11.5 \pm 2$ & $<0.05$ \\
\hline Color tissue Doppler S wave (cm/s) & $4.8 \pm 2.3$ & $8.5 \pm 3.5$ & 0.06 \\
\hline dP/dT (mmHg/s) & $320 \pm 45$ & $390 \pm 53$ & \\
\hline $\begin{array}{l}\text { FAC }- \text { fractional area change, RIMP - right ventricular index of myocardial performance, RV }- \text { right } \\
\text { ventricle, SD - standard deviation, TAPSE - tricuspid annulus peak systolic velocity, RVEF }- \text { right } \\
\text { ventricle ejection fraction; KPN - ketogenic parenteral nutrition. }\end{array}$ & & \\
\hline
\end{tabular}

\section{Figures}




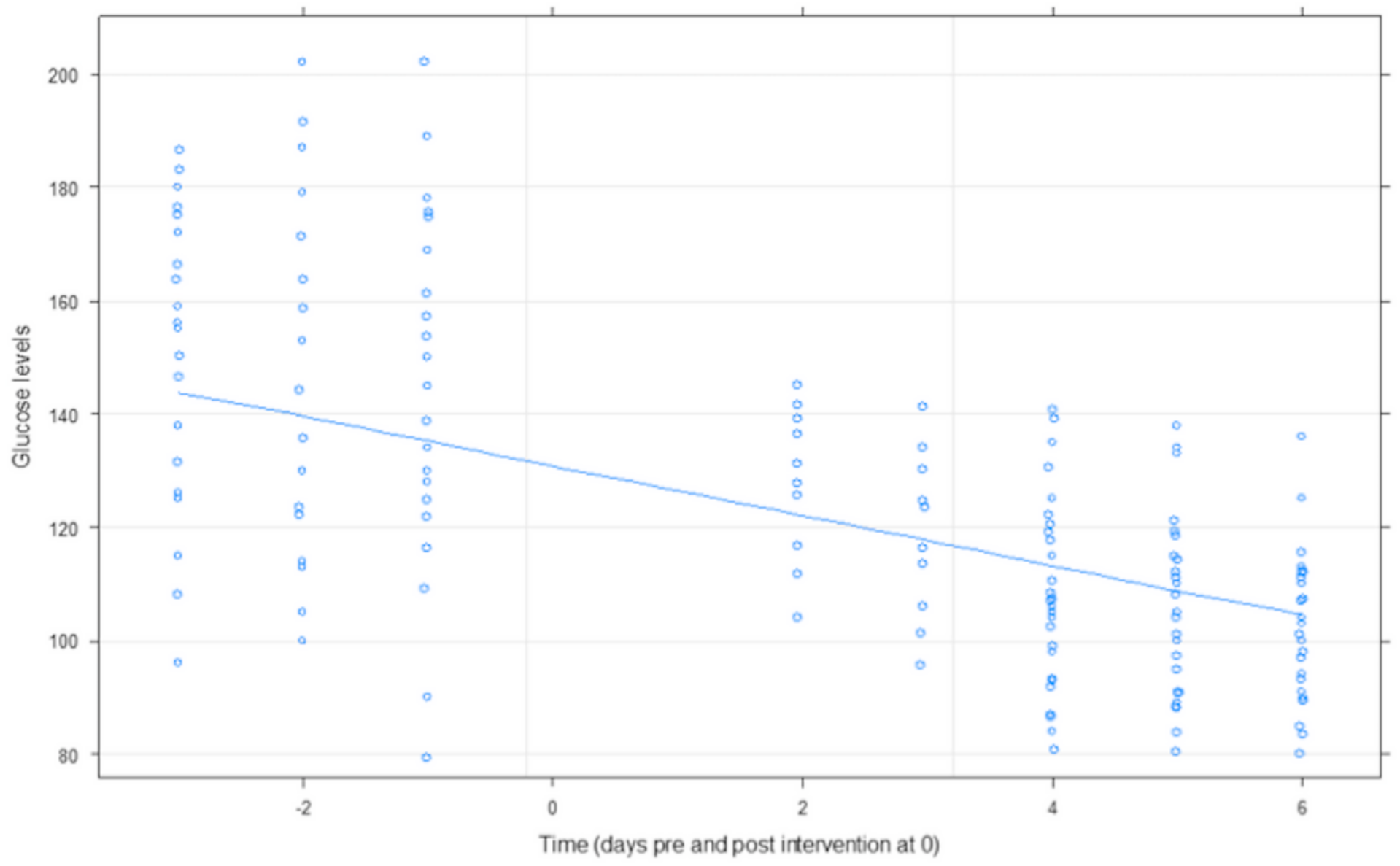

Figure 1

Glucose levels in pre and post KPN treatment period. p-value for change in trend at day " 0 " 0.45 . Overall linear trend $p$-value: $<0.001$. (Glucose levels $\mathrm{mg} / \mathrm{dl}$ ) 


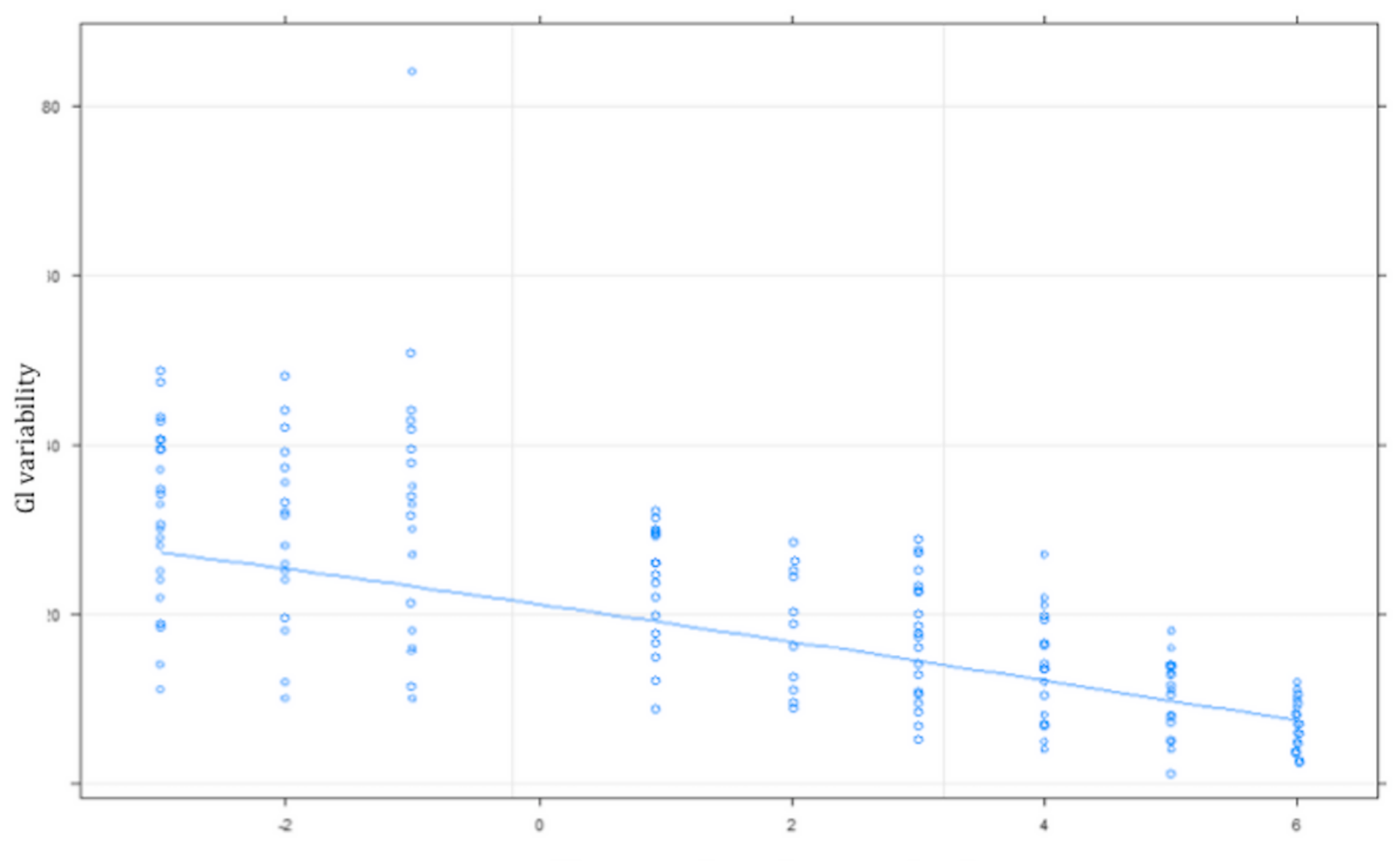

Time pre and post therapy at time 0

\section{Figure 2}

Trend of glycaemia variability in pre and post KPN treatment period. p-value for change in trend at day " 0 " 0.035 . Overall linear trend $p$-value: $<0.001$. (Glucose levels $\mathrm{mg} / \mathrm{dl}$ ) 


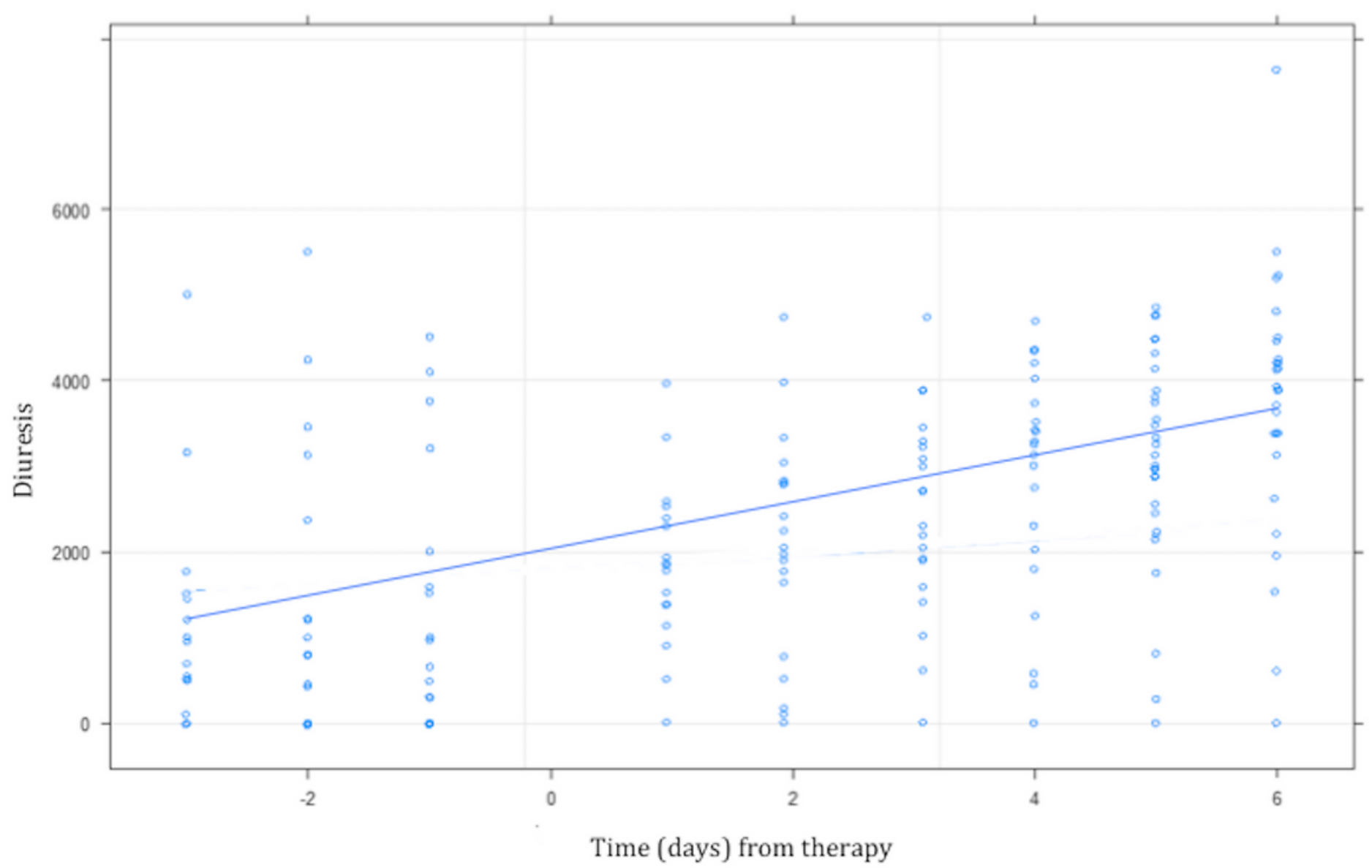

Figure 3

Trend of urine output volume in pre and post KPN treatment period. p-value for change in trend at day " 0 " 0.98. Overall linear trend $\mathrm{p}$-value: 0.36 . (Diuresis $\mathrm{ml} / \mathrm{day}$ )

\section{Supplementary Files}

This is a list of supplementary files associated with this preprint. Click to download.

- GraphicAbstractLipidinSugarOut1.jpg 